\title{
Predicate-Aware, Makespan-Preserving Software Pipelining of Scheduling Tables
}

THOMAS CARLE and DUMITRU POTOP-BUTUCARU, INRIA Paris-Rocquencourt

We propose a software pipelining technique adapted to specific hard real-time scheduling problems. Our technique optimizes both computation throughput and execution cycle makespan, with makespan being prioritary. It also takes advantage of the predicated execution mechanisms of our embedded execution platform. To do so, it uses a reservation table formalism allowing the manipulation of the execution conditions of operations. Our reservation tables allow the double reservation of a resource at the same dates by two different operations, if the operations have exclusive execution conditions. Our analyses can determine when double reservation is possible even for operations belonging to different iterations.

Categories and Subject Descriptors: D.3.4 [Programming Languages]: Processors-Compilers; D.3.4 [Programming Languages]: Processors-Optimization; C.3 [Special-Purpose and Application-Based Systems]: Real-Time and Embedded Systems

General Terms: Algorithms, Performance

Additional Key Words and Phrases: Bi-criterion optimization, Predicate-aware, Real-time scheduling, Scheduling table, Software pipelining

ACM Reference Format:

Thomas Carle and Dumitru Potop-Butucaru. 2014. Predicate-aware, makespan-preserving software pipelining of scheduling tables. ACM Trans. Architec. Code Optim. 11, 1, Article 12 (February 2014), 26 pages.

DOI: http://dx.doi.org/10.1145/2579676

\section{INTRODUCTION}

In this article, we take inspiration from a classical compilation technique, namely software pipelining, in order to improve the system-level task scheduling of a specific class of embedded systems.

Software pipelining. Compilers are expected to improve code speed by taking advantage of microarchitectural instruction-level parallelism [Hennessy and Patterson 2007]. Pipelining compilers usually rely on reservation tables to represent an efficient (possibly optimal) static allocation of the computing resources (execution units and/or registers) with a timing precision equal to that of the hardware clock. Executable code is then generated that enforces this allocation, possibly with some timing flexibility. But on VLIW architectures, where each instruction word may start several operations, this flexibility is very limited, and generated code is virtually identical to the reservation table. The scheduling burden is mostly supported here by the compilers, which

This work was partially funded by the FUI PARSEC project. Preliminary results of this work are also available online as the Chalmers' master thesis of the first author (http://publications.lib.chalmers.se/records/ fulltext/146444.pdf).

Authors' addresses: Thomas Carle, Domaine de Voluceau, BP 105, 78153 Le Chesnay, Cedex, France; email: thomas.carle@inria.fr; Dumitru Potop-Butucaru, INRIA Paris-Rocquencourt, Domaine de Voluceau - Rocquencourt, BP 105, 78153 Le Chesnay, Cedex, France; email: dumitru.potop@inria.fr.

Permission to make digital or hard copies of part or all of this work for personal or classroom use is granted without fee provided that copies are not made or distributed for profit or commercial advantage and that copies show this notice on the first page or initial screen of a display along with the full citation. Copyrights for components of this work owned by others than ACM must be honored. Abstracting with credit is permitted. To copy otherwise, to republish, to post on servers, to redistribute to lists, or to use any component of this work in other works requires prior specific permission and/or a fee. Permissions may be requested from Publications Dept., ACM, Inc., 2 Penn Plaza, Suite 701, New York, NY 10121-0701 USA, fax +1 (212) 869-0481, or permissions@acm.org.

(c) 2014 ACM 1544-3566/2014/02-ART12 $\$ 15.00$

DOI: http://dx.doi.org/10.1145/2579676 
include software pipelining techniques [Rau and Glaeser 1981; Allan et al. 1995] designed to increase the throughput of loops by allowing one loop cycle to start before the completion of the previous one.

Static (offline) real-time scheduling. A very similar picture can be seen in the systemlevel design of safety-critical real-time embedded control systems with distributed (parallel, multicore) hardware platforms. The timing precision is here coarser, both for starting dates, which are typically given by timers, and for durations, which are characterized with Worst-Case Execution Times (WCETs). However, safety and efficiency arguments [Fohler et al. 2008] lead to the increasing use of tightly synchronized time-triggered architectures and execution mechanisms, defined in well-established standards such as TTA, FlexRay [Rushby 2001], ARINC653 [ARINC653 2005], or AUTOSAR [AUTOSAR 2009]. Systems based on these platforms typically have hard realtime constraints, and their correct functioning must be guaranteed by a schedulability analysis. In this article, we are interested in statically scheduled systems where resource allocation can be described under the form of a reservation/scheduling table that constitutes, by itself, a proof of schedulability. Such systems include:

-Periodic time-triggered systems [Caspi et al. 2003; Zheng et al. 2005; Monot et al. 2010; Eles et al. 2000; Potop-Butucaru et al. 2010] that are naturally mapped over ARINC653, AUTOSAR, TTA, or FlexRay.

- Systems where the scheduling table describes the reaction to some sporadic input event (meaning that the table must fit inside the period of the sporadic event). Such systems can be specified in AUTOSAR, allowing, for instance, the modeling of computations synchronized with engine rotation events [André et al. 2007].

- Some systems with a mixed event-driven/time-driven execution model, such as those synthesized by SynDEx [Grandpierre and Sorel 2003].

Synthesis of such systems starts from specifications written in domain-specific formalisms such as Simulink or SCADE [Caspi et al. 2003]. These formalisms allow the description of concurrent data computations and communications that are conditionally activated at each cycle of the embedded control algorithm depending on the current input and state of the system.

The problem. The optimal scheduling of such specifications onto platforms with multiple, heterogenous execution and communication resources (distributed, parallel, multicore) is NP-hard regardless of the optimality criterion (throughput, makespan, etc.) [Garey and Johnson 1979]. Existing scheduling techniques and tools [Caspi et al. 2003; Zheng et al. 2005; Grandpierre and Sorel 2003; Potop-Butucaru et al. 2010; Eles et al. 2000] heuristically solve the simpler problem of synthesizing a scheduling table of minimal length, which implements one generic cycle of the embedded control algorithm. In a hard real-time context, minimizing table length (i.e., the makespan, as defined later in the glossary of Figure 2) is often a good idea, because in many applications it bounds the response time after a stimulus.

But optimizing makespan alone relies on an execution model where execution cycles cannot overlap in time (no pipelining is possible), even if resource availability allows it. At the same time, most real-time applications have both makespan and throughput requirements, and in some cases achieving the required throughput is only possible if a new execution cycle is started before the previous one has completed.

This is the case in the Electronic Control Units (ECUs) of combustion engines. Starting from the acquisition of data for a cylinder in one engine cycle, an ECU must compute the ignition parameters before the ignition point of the same cylinder in the next engine cycle (a makespan constraint). It must also initiate one such computation for each cylinder in each engine cycle (a throughput constraint). On modern multiprocessor ECUs, meeting both constraints requires the use of pipelining [André et al. 


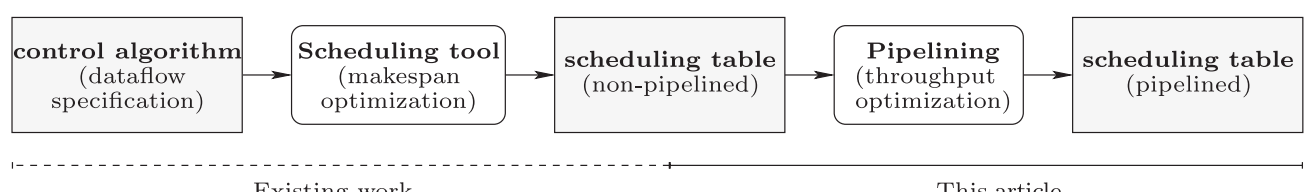

Existing work

This article

Fig. 1. Proposed pipelined scheduling flow.

\begin{tabular}{|c|c|}
\hline Concept & Description \\
\hline scheduling table & $\begin{array}{l}\text { These are essentially modulo reservation tables [Lam 1988], ex- } \\
\text { tended to allow the representation of conditional/multiple reser- } \\
\text { vations. We use a different name to identify our specific internal } \\
\text { formalism where reservations are the complex scheduled opera- } \\
\text { tions defined below. }\end{array}$ \\
\hline initiation interval & $\begin{array}{l}\text { The length of a schedule table is also called its initiation interval } \\
\text { (II). }\end{array}$ \\
\hline execution cycle & $\begin{array}{l}\text { One iteration of either a scheduling table or the control algorithm } \\
\text { before scheduling. }\end{array}$ \\
\hline $\begin{array}{l}\text { nonpipelined } \\
\text { pipelined }\end{array}$ & $\begin{array}{l}\text { In classical software pipelining, reservation tables are used to rep- } \\
\text { resent the pipelined schedule. In our case, a reservation table } \\
\text { is also used to represent the input of the pipelining algorithm. } \\
\text { To avoid ambiguity, uses of "scheduling table," "initiation inter- } \\
\text { val," "throughput," etc., will be qualified with "nonpipelined" } \\
\text { or "pipelined" whenever necessary. For instance, the pipelined } \\
\text { scheduling table is usually known in software pipelining as the } \\
\text { kernel. }\end{array}$ \\
\hline makespan & $\begin{array}{l}\text { Worst-case duration of one execution cycle of the control algo- } \\
\text { rithm, from the start of its first operation to the end of its last } \\
\text { operation. In our approach, by construction, it is the same in both } \\
\text { the nonpipelined and pipelined scheduling tables. It is equal to } \\
\text { the initiation interval of the nonpipelined scheduling table. }\end{array}$ \\
\hline throughput & $\begin{array}{l}\text { The number of execution cycles of the control algorithm executed } \\
\text { per time unit. It is defined as the inverse of the initiation interval. }\end{array}$ \\
\hline $\begin{array}{l}\text { intercycle depen- } \\
\text { dency }\end{array}$ & $\begin{array}{l}\text { Data dependencies between operations of different execution cy- } \\
\text { cles of the nonpipelined scheduling table. }\end{array}$ \\
\hline $\begin{array}{l}\text { scheduled opera- } \\
\text { tion }\end{array}$ & $\begin{array}{l}\text { Complex data structure defining a resource reservation in a } \\
\text { scheduling table (a time interval on one or several resources), but } \\
\text { providing at the same time information on the operation (real- } \\
\text { time task) to be executed inside this reservation (input and output } \\
\text { variables, execution condition). }\end{array}$ \\
\hline
\end{tabular}

Fig. 2. Glossary of terms used in this article. All notions are formally defined later.

2007]. Another example is that of systems where a faster rate of sensor data acquisition results in better precision and improved control, but optimizing this rate must not lead to the nonrespect of requirements on the latency between sensor acquisition and actuation. To allow the scheduling of such systems, we consider here the static scheduling problem of optimizing both makespan and throughput, with makespan being prioritary.

Contribution. To (heuristically) solve this optimization problem, we use a two-phase scheduling flow that can be seen as a form of decomposed software pipelining [Wang and Eisenbeis 1993; Gasperoni and Schwiegelshohn 1994; Calland et al. 1998]. As pictured in Figure 1, the first phase of this flow consists in applying one of the previously mentioned makespan-optimizing tools. The result is a scheduling table describing the execution of one generic execution cycle of the embedded control algorithm with no pipelining. 
The second phase uses a novel software pipelining algorithm, introduced in this article, to significantly improve the throughput without changing the makespan and while preserving the periodic nature of the system. The approach has the advantage of simplicity and generality, allowing the use of existing makespan-optimizing tools.

The proposed software pipelining algorithm is a very specific and constrained form of modulo scheduling [Rau 1996]. Like all modulo scheduling algorithms, it determines a shorter initiation interval for the execution cycles (iterations) of the control algorithm, subject to resource and intercycle data dependency constraints. Unlike previous modulo scheduling algorithms, however, it starts from an already scheduled code (the nonpipelined scheduling table) and preserves all of the intracycle scheduling decisions made at phase 1 in order to preserve the makespan unchanged. In other words, our algorithm computes the best initiation interval for the nonpipelined scheduling table and reorganizes resource reservations into a pipelined scheduling table, whose length is equal to the new initiation interval, and which accounts for the changes in memory allocation.

We have implemented our algorithm into a pipelining tool that is used, as we desired, in conjunction with an existing makespan-optimizing scheduling tool. The resulting two-phase flow gives good results on architectures without temporal partitioning [ARINC653 2005], like the previously mentioned AUTOSAR or SynDEx-generated applications and, to a certain extent, applications using the FlexRay dynamic segment.

For applications mapped onto partitioned architectures (ARINC 653, TTA, or FlexRay, the static segment) or where the nonfunctional specification includes multiple release date, end-to-end latency, or periodicity constraints, separating the implementation process in two phases (scheduling followed by pipelining) is not a good idea. We therefore developed a single-phase pipelined scheduling technique documented elsewhere [Carle et al. 2012] but that uses (with good results) the same internal representation based on scheduling tables to allow a simple mapping of applications with execution modes onto heterogenous architectures with multiple processors and buses.

\section{Outline}

The remainder of the article is structured as follows. Section 2 reviews related work. Section 3 formally defines scheduling tables. Section 4 presents the pipelining technique and provides a complex example. Section 5 deals with data dependency analysis. Section 6 gives experimental results, and Section 7 concludes.

\section{RELATED WORK AND ORIGINALITY}

This section reviews related work and details the originality points of our work. Performing this comparison required us to relate concepts and techniques belonging to two fields: software pipelining and real-time scheduling. To avoid ambiguities when the same notion has different names depending on the field, in Figure 2 we present a glossary of terms that will be used throughout the article.

\subsection{Decomposed Software Pipelining}

Closest to our work are previous results on decomposed software pipelining [Wang and Eisenbeis 1993; Gasperoni and Schwiegelshohn 1994; Calland et al. 1998]. In these papers, the software pipelining of a sequential loop is realized using two-phase heuristic approaches with good practical results. Two main approaches are proposed in these papers.

In the first approach, used in all three cited papers, the first phase consists in solving the loop scheduling problem while ignoring resource constraints. As noted in Calland et al. [1998], existing decomposed software pipelining approaches solve this loop scheduling problem by using retiming algorithms. Retiming [Leiserson and Saxe 
1991] can therefore be seen as a very specialized form of pipelining targeted at cyclic (synchronous) systems where each operation has its own execution unit. Retiming has significant restrictions when compared with full-fledged software pipelining:

-It is oblivious to resource allocation. As a side effect, it cannot take into account execution conditions to improve allocation, being defined in a purely dataflow context.

-It requires that the execution cycles of the system do not overlap in time so that one operation must be completely executed inside the cycle where it was started.

Retiming can only change the execution order of the operations inside an execution cycle. A typical retiming transformation is to move one operation from the end to the beginning of the execution cycle in order to shorten the duration (critical path) of the execution cycle and thus improve system throughput. The transformation cannot decrease the makespan but may increase it.

Once retiming is done, the second transformation phase takes into account resource constraints. To do so, it considers the acyclic code of one generic execution cycle (after retiming). A list scheduling technique ignoring intercycle dependences is used to map this acyclic code (which is actually represented with a Directed Acyclic Graph [DAG]) over the available resources.

The second technique for decomposed software pipelining, presented in Wang and Eisenbeis [1993], basically switches the two phases presented earlier. Resource constraints are considered here in the first phase, through the same technique used earlier: list scheduling of DAGs. The DAG used as input is obtained from the cyclic loop specification by preserving only some of the data dependences. This scheduling phase decides the resource allocation and the operation order inside an execution cycle. The second phase takes into account the data dependences that were discarded in the first phase. It basically determines the fastest way that a specification-level execution cycle can be executed by several successive pipelined execution cycles without changing the operation scheduling determined in phase 1 (preserving the throughput unchanged). Minimizing the makespan is important here because it results in a minimization of the memory/register use.

\subsection{Originality}

In this article, we propose a third decomposed software pipelining technique with two significant originality points, detailed next.

2.2.1. Optimization of Both Makespan and Throughput. Existing software pipelining techniques are tailored for optimizing only one real-time performance metric: the processing throughput of loops [Yun et al. 2003] (sometimes besides other criteria such as register usage [Govindarajan et al. 1994; Zalamea et al. 2004; Huff 1993] or code size [Zhuge et al. 2002]). In addition to throughput, we also seek to optimize makespan, with makespan being prioritary. Recall that throughput and latency (makespan) are antagonistic optimization objectives during scheduling [Benoît et al. 2007], meaning that resulting schedules can be quite different (an example will be provided in Section 4.1.2).

To optimize makespan, in the first phase of our approach, we employ existing scheduling techniques that were specifically designed for this purpose [Caspi et al. 2003; Zheng et al. 2005; Grandpierre and Sorel 2003; Potop-Butucaru et al. 2010; Eles et al. 2000]. But the main contribution of this article concerns the second phase of our flow, which takes the scheduling table computed in phase 1 and optimizes its throughput while keeping its makespan unchanged. This is done using a new algorithm that conserves all of the allocation and intracycle scheduling choices made in phase 1 (thus conserving 
makespan guarantees) but allowing the optimization of the throughput by increasing (if possible) the frequency with which execution cycles are started.

Like retiming, this transformation is a very restricted form of modulo scheduling software pipelining. In our case, it can only change the initiation interval (changes in memory allocation and in the scheduling table are only consequences). By comparison, classical software pipelining algorithms, such as the iterative modulo scheduling of Rau [1996], perform a full mapping of the code involving both execution unit allocation and scheduling. Our choice of transformation is motivated by three factors:

-It preserves makespan guarantees.

-It gives good practical results for throughput optimization.

-It has low complexity.

It is important to note that our transformation is not a form of retiming. Indeed, it allows for a given operation to span over several cycles of the pipelined implementation, and it can take advantage of conditional execution to improve pipelining, whereas retiming techniques work in a pure dataflow context, without predication (an example will be provided in Section 4.1.2).

2.2.2. Predication. For an efficient mapping of our conditional specifications, it is important to allow an independent, predicated (conditional) control of the various computing resources. However, most existing techniques for software pipelining [Allan et al. 1995; Warter et al. 1993; Yun et al. 2003] use hardware models that significantly constrain or simply prohibit predicated resource control. This is due to limitations in the target hardware itself. One common problem is that two different operations cannot be scheduled at the same date on a given resource (functional unit), even if they have exclusive predicates (like the branches of a test). The only exception we know to this rule is Predicate-Aware Scheduling (PAS) [Smelyanskyi et al. 2003].

By comparison, the computing resources of our target architectures are not a mere functional units of a CPU (as in classical predicated pipelining), but full-fledged processors such as PowerPC and ARM. The operations executed by these computing resources are large sequential functions and not simple CPU instructions. Thus, each computing resource allows unrestricted predication control by means of conditional instructions, and the timing overhead of predicated control is negligible with respect to the duration of the operations. This means that our architectures satisfy the PAS requirements. The drawback of PAS is that sharing the same resource at the same date is only possible for operations of the same cycle, due to limitations in the dependency analysis phase. Our technique removes this limitation (an example will be provided in Section 4.1.3).

To exploit the full predicated control of our platform, we rely on a new intermediate representation, namely predicated and pipelined scheduling tables. By comparison to the modulo reservation tables of Lam [1988] and Rau [1996], our scheduling tables allow the explicit representation of the execution conditions (predicates) of the operations. In turn, this allows the double reservation of a given resource by two operations with exclusive predicates.

\subsection{Other Aspects}

A significant amount of work exists on applying software pipelining or retiming techniques for the efficient scheduling of tasks onto coarser-grain architectures, such as multiprocessors [Kim et al. 2012; Yang and Ha 2009; Chatha and Vemuri 2002; Chiu et al. 2011; Caspi et al. 2003; Morel 2005]. To our best knowledge, these results share the two fundamental limitations of other software pipelining algorithms: optimizing for only one real-time metric (throughput) and not fully taking advantage of conditional execution to allow double allocation of resources. 


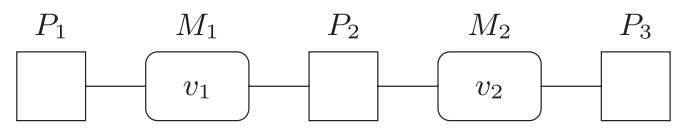

Fig. 3. Simple architecture.

Minor originality points of our technique, concerning code generation and dependency analysis, will be discussed and compared with previous work in Sections 4.4 and 5.

\section{SCHEDULING TABLES}

This section defines the formalism used to represent the nonpipelined static schedules produced by phase 1 of our scheduling flow and taken as input by phase 2 . Inspired by Potop-Butucaru et al. [2010] and Grandpierre and Sorel [2003], our scheduling table formalism remains at a significantly lower abstraction level. The models of PotopButucaru et al. [2010] and Grandpierre and Sorel [2003] are fully synchronous: each variable has at most one value at each execution cycle, and moving one value from a cycle to the next can only be done through explicit delay constructs. In our model, each variable (called a memory cell) can be assigned several times during a cycle, and values are by default passed from one execution cycle to the next.

The lower abstraction level means that time-triggered executable code generated by any of the previously mentioned scheduling tools can directly be used as input for the pipelining phase. Integration between the scheduling tools and the pipelining algorithm defined next is thus facilitated. The downside is that the pipelining technique is more complex, as detailed in Section 4.4.2.

\subsection{Architecture Model}

We model our multiprocessor (distributed, parallel) execution architectures using a very simple language defining sequential execution resources, memory blocks, and their interconnections. Formally, an architecture model is a bipartite undirected graph $\mathcal{A}=<$ $\mathcal{P}, \mathcal{M}, \mathcal{C}>$, with $\mathcal{C} \subseteq \mathcal{P} \times \mathcal{M}$. The elements of $\mathcal{P}$ are called processors, but they model all of the computation and communication devices capable of independent execution (CPU cores, accelerators, DMA, and bus controllers, etc.). We assume that each processor can execute only one operation at a time. We also assume that each processor has its own sequential or time-triggered program. This last assumption is natural on actual CPU cores. On devices such as DMAs and accelerators, it models the assumption that the cost of control by some other processor is negligible. ${ }^{1}$

The elements of $\mathcal{M}$ are RAM blocks. We assume that each RAM block is structured as a set of disjoint cells. We denote with Cells the set of all memory cells in the system, and with Cell $_{M}$ the set of cells on RAM block $M$. Our model does not explicitly specify memory size limits. To limit memory usage in the pipelined code, we rely instead on the mechanism detailed in Section 4.4.3.

The elements of $\mathcal{C}$ are the interconnections. Processor $P$ has direct access to memory block $M$ whenever $(P, M) \in \mathcal{C}$. All processors directly connected to a memory block $M$ can access $M$ at the same time. Therefore, care must be taken to prohibit concurrent read-write or write-write access by two or more processors to a single memory cell, in order to preserve functional determinism (we will assume that this is ensured by the input scheduling table and will be preserved by the pipelined one).

The simple architecture of Figure 3 has three processors $\left(P_{1}, P_{2}\right.$, and $\left.P_{3}\right)$ and two memory blocks $\left(M_{1}\right.$ and $\left.M_{2}\right)$. Each of the $M_{i}$ blocks has only one memory cell $v_{i}$.

\footnotetext{
${ }^{1}$ This assumption is common for coarse-grain real-time applications.
} 


\subsection{Scheduling Tables}

On such architectures, phase 1 scheduling algorithms perform static (offline) allocation and scheduling of embedded control applications under a periodic execution model. The result is represented with scheduling tables, which are finite time-triggered activation patterns. This pattern defines the computation of one period (also called execution cycle) of the control algorithm. The infinite execution of the embedded system is the infinite succession of periodically triggered execution cycles. Execution cycles do not overlap in time (there is no pipelining).

Formally, a scheduling table is a triple $\mathcal{S}=<p, \mathcal{O}$, Init $>$, where $p$ is the activation period of execution cycles, $\mathcal{O}$ is the set of scheduled operations, and Init is the initial state of the memory.

The activation period gives the (fixed) duration of the execution cycles. All operations of one execution cycle must be completed before the following execution cycle starts. The activation period thus sets the length of the scheduling table and is denoted by $\operatorname{len}(\mathcal{S})$.

The set $\mathcal{O}$ defines the operations of the scheduling table. Each scheduled operation $o \in \mathcal{O}$ is a tuple defining:

$-\operatorname{In}(o) \subseteq$ Cells is the set of memory cells read by $o$.

$-O u t(o) \subseteq$ Cells is the set of cells written by $o$.

- Guard $(o)$ is the execution condition of $o$, defined as a predicate over the values of memory cells.

-We denote with GuardIn(o) the set of memory cells used in the computation of Guard(o). There is no relation between GuardIn(o) and $\operatorname{In}(o)$.

$-\operatorname{Res}(o) \subseteq \mathcal{P}$ is the set of processors used during the execution of $o$.

$-t(o)$ is the start date of $o$.

$-d(o)$ is the duration of $o$. The duration is viewed here as a time budget the operation must not exceed. This can be statically ensured through a WCET analysis.

All of the resources of $\operatorname{Res}(o)$ are exclusively used by $o$ after $t(o)$ and for a duration of $d(o)$ in cycles where Guard (o) is true. The sets $\operatorname{In}(o)$ and Out(o) are not necessarily disjoint, to model variables that are both read and updated by an operation. For lifetime analysis purposes, we assume that input and output cells are used for the duration of the operation. The cells of GuardIn(o) are all read at the beginning of the operation, but we assume that the duration of the computation of the guard is negligible (zero time). ${ }^{2}$

To cover cases where a memory cell is used by one operation before being updated by another, each memory cell can have an initial value. For a memory cell $m$, Init $(m)$ is either nil or some constant.

3.2.1. A Simple Example. To exemplify, we consider the simple dataflow synchronous specification of Figure 4(a), which we map onto the architecture of Figure 3. Depending on the nonfunctional requirements given as input to the scheduling tool of phase 1 (allocation constraints, WCETs, etc.), one possible result is the scheduling table pictured in Figure 4. We assumed here that $A$ is must be mapped onto $P_{1}$ (e.g., because it uses a sensor peripheral connected to $P_{1}$ ); that $B$ must be mapped onto $P_{2}$; that $C$ must be mapped onto $P_{3}$; and that $A, B$, and $C$ have all duration 1 .

This table has a length of 3 and contains the three operations of the dataflow specification $(A, B$, and $C)$. Operation $A$ reads no memory cell but writes $v_{1}$ so that $\operatorname{In}(A)=\emptyset$ and $\operatorname{Out}(A)=\left\{v_{1}\right\}$. Similarly, $\operatorname{In}(B)=\left\{v_{1}\right\}, \operatorname{Out}(B)=\operatorname{In}(C)=\left\{v_{2}\right\}$, and $\operatorname{Out}(C)=\varnothing$.

${ }^{2}$ The memory access model where an operation reads its inputs at start time, writes its outputs upon completion, and where guard computations take time can be represented on top of our model. 


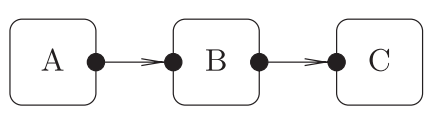

(a)

\begin{tabular}{r|c|c|c|}
\multirow{2}{*}{ time } & \multicolumn{3}{|c|}{ Processor } \\
\cline { 2 - 4 } & P1 & P2 & P3 \\
\hline 0 & A@true & & \\
\cline { 2 - 3 } 1 & & B@true & \\
\cline { 3 - 4 } 2 & & & C@true \\
\hline
\end{tabular}

(b)

Fig. 4. Simple dataflow specification (a) and (nonpipelined) scheduling table for this specification (b).

All three operations are executed at every cycle, so their guard is true (guards are graphically represented with "@true"). The three operations are each allocated on one processor: $\operatorname{Res}(A)=\left\{P_{1}\right\}, \operatorname{Res}(B)=\left\{P_{2}\right\}, \operatorname{Res}(C)=\left\{P_{3}\right\}$. Finally, $t(A)=0, t(B)=1$, $t(C)=2$, and $d(A)=d(B)=d(C)=1$. No initialization of the memory cells is needed (the initial values are all $n i l$ ).

\subsection{Well-Formed Properties}

The formalism presented provides the syntax of scheduling tables and allows the definition of operational semantics. However, not all syntactically correct tables represent correct implementations. Some of them are nondeterministic due to data races or due to operations exceeding their time budgets. Others are simply unimplementable, for instance because an operation is scheduled on processor $P$ but accesses memory cells on a RAM block not connected to $P$. A set of correctness properties is therefore necessary to define the well-formed scheduling tables.

However, some of these properties are not important in this article, because we assume that the input of our pipelining technique, synthesized by a scheduling tool, is already correct. For instance, we assume that all schedules are implementable, with data used by a processor being allocated in adjacent memory banks. This is why we only formalize here two correctness properties that will need attention in the following sections because pipelining transformations can affect them.

We say that two operations $o_{1}$ and $o_{2}$ are nonconcurrent, denoted $o_{1} \perp o_{2}$, if either their executions do not overlap in time $\left(t\left(o_{1}\right)+d\left(o_{1}\right) \leq t\left(o_{2}\right)\right.$ or $\left.t\left(o_{2}\right)+d\left(o_{2}\right) \leq t\left(o_{1}\right)\right)$, or if they have exclusive guards $\left(\operatorname{Guard}\left(o_{1}\right) \wedge \operatorname{Guard}\left(o_{2}\right)=\right.$ false). With this notation, the following correctness properties are assumed respected by input (nonpipelined) scheduling tables and must be respected by the output (pipelined) ones:

Sequential processors. No two operations can use a processor at the same time. Formally, for all $o_{1}, o_{2} \in \mathcal{O}$, if $\operatorname{Res}\left(o_{1}\right) \cap \operatorname{Res}\left(o_{2}\right) \neq \emptyset$, then $o_{1} \perp o_{2}$.

No data races. If some memory cell $m$ is written by $o_{1}\left(m \in\right.$ Out $\left.\left(o_{1}\right)\right)$ and is used by $o_{2}\left(m \in \operatorname{In}\left(o_{2}\right) \cup \operatorname{Out}\left(o_{2}\right)\right)$, then $o_{1} \perp o_{2}$.

\section{PIPELINING TECHNIQUE OVERVIEW}

\subsection{Pipelined Scheduling Tables}

4.1.1. A Simple Example. For the example in Figure 4, an execution where successive cycles do not overlap in time is clearly suboptimal. Our objective is to allow the pipelined execution of Figure 5, which ensures a maximal use of the computing resources.

In the pipelined execution, a new instance of operation $A$ starts as soon as the previous one has completed, and the same is true for $B$ and $C$. The first two time units of the execution are the prologue, which fills the pipeline. In the steady state, the pipeline is full and has a throughput of one computation cycle (of the nonpipelined 


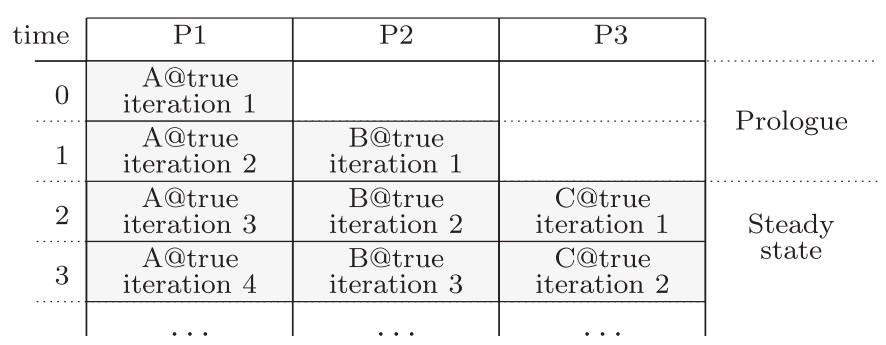

Fig. 5. Pipelined execution trace for Figure 4.

\begin{tabular}{r|c|c|c|}
\cline { 2 - 4 } time & $\mathrm{P} 1$ & $\mathrm{P} 2$ & $\mathrm{P} 3$ \\
\hline 0 & $\mathrm{~A} @$ true & $\mathrm{B} @$ true & $\mathrm{C} @$ true \\
$f$ st $(A)=0$ & $f s t(B)=1$ & $f s t(C)=2$ \\
\hline
\end{tabular}

Fig. 6. Pipelined scheduling table (kernel) for Figure 4.

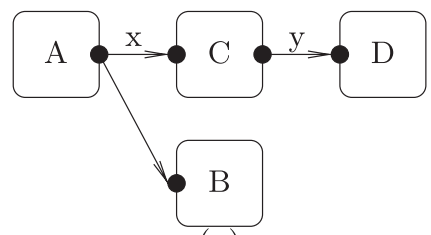

(a)

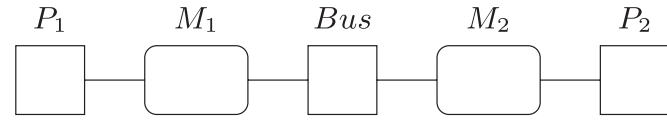

(b)

Fig. 7. Example 2: Dataflow specification (a) and a bus-based implementation architecture (b).

system) per time unit. If the system is allowed to terminate, then completion is realized by the epilogue, not pictured in our example, which empties the pipeline.

We represent the pipelined system schedule using the pipelined scheduling table pictured in Figure 6. Its length is 1, corresponding to the throughput of the pipelined system. The operation set contains the same operations $A, B$, and $C$, but there are significant changes. The start dates of $B$ and $C$ are now 0 , as the three operations are started at the same time in each pipelined execution cycle. A nonpipelined execution cycle spans over several pipelined cycles, and each pipelined cycle starts one nonpipelined cycle.

To account for the prologue phase, where operations progressively start to execute, each operation is assigned a start index $f s t(o)$. If an operation $o$ has $f s t(o)=n$, it will first be executed in the pipelined cycle of index $n$ (indices start at 0 ). Due to pipelining, the instance of $o$ executed in the pipelined cycle $m$ belongs to the nonpipelined cycle of index $m-f s t(o)$. For instance, operation $C$ with $f s t(C)=2$ is first executed in the third pipelined cycle (of index 2) but belongs to the first nonpipelined cycle (of index 0 ).

4.1.2. Example 2: Makespan vs. Throughput Optimization. Figure 7 showcases how the different optimization objectives of our technique lead to different scheduling results when compared with existing software pipelining techniques.

The architecture here is more complex, involving a communication bus that connects the two identical processors. Communications over the bus are synthesized during the scheduling process, as needed. Note that the bus is modeled as a processor performing communication operations. This level of description, defined in Section 3.1, is accurate enough to support our pipelining algorithms. The makespan-optimizing scheduling algorithms used in phase 1 use more detailed architecture descriptions (outside the scope of this article). 


\begin{tabular}{|c|c|c|c|}
\hline time & $\mathrm{P} 1$ & Bus & $\mathrm{P} 2$ \\
\hline 0 & A@true & & \\
\hline 1 & \multirow{2}{*}{ B@true } & $\mathrm{x} @$ true & \\
\hline 2 & & & \multirow{4}{*}{$\mathrm{C} @$ true } \\
\hline 3 & & & \\
\hline 4 & & & \\
\hline 5 & & & \\
\hline 6 & & & D@true \\
\hline
\end{tabular}

(a)

\begin{tabular}{|c|c|c|c|}
\hline time & $\mathrm{P} 1$ & Bus & $\mathrm{P} 2$ \\
\hline 0 & $\begin{array}{c}\mathrm{A} @ \text { true } \\
\text { fst }(\mathrm{A})=0\end{array}$ & & $\begin{array}{c}\text { C@true } \\
\text { fst }(\mathrm{C})=1\end{array}$ \\
\hline 1 & \multirow{2}{*}{$\begin{array}{c}B @ \text { true } \\
\text { fst }(B)=0\end{array}$} & $\begin{array}{c}x @ \operatorname{true} \\
\text { fst }(\text { SND })=0\end{array}$ & $\begin{array}{c}\text { D@true } \\
\text { fst }(D)=1\end{array}$ \\
\hline 2 & & & \multirow{3}{*}{$\begin{array}{c}\text { C@true } \\
\text { fst }(\mathrm{C})=0\end{array}$} \\
\hline 3 & & & \\
\hline 4 & & & \\
\hline
\end{tabular}

(b)

Fig. 8. Example 2: Nonpipelined scheduling table produced by phase 1 of our technique (a) and pipelined table produced by phase 2 (b) for Figure 7.

\begin{tabular}{|c|c|c|c|}
\hline time & $\mathrm{P} 1$ & Bus & $\mathrm{P} 2$ \\
\hline 0 & $\begin{array}{c}\mathrm{A} @ \text { true } \\
\text { fst }(\mathrm{A})=0\end{array}$ & & $\begin{array}{c}D @ \operatorname{true} \\
\text { fst }(\mathrm{D})=1\end{array}$ \\
\hline 1 & \multirow{2}{*}{$\begin{array}{c}\text { B@true } \\
\text { fst }(B)=0\end{array}$} & $\begin{array}{c}x @ \operatorname{true} \\
\text { fst }(\text { SND })=0\end{array}$ & \\
\hline 2 & & & \multirow{4}{*}{$\begin{array}{c}\text { C@true } \\
\text { fst }(C)=0\end{array}$} \\
\hline 3 & & & \\
\hline 4 & & & \\
\hline 5 & & & \\
\hline
\end{tabular}

(a)

\begin{tabular}{|c|c|c|c|}
\hline time & $\mathrm{P} 1$ & Bus & P2 \\
\hline 0 & $\begin{array}{c}\text { A@true } \\
\text { fst }(A)=0\end{array}$ & & \multirow{2}{*}{$\begin{array}{c}\text { C@true } \\
\text { fst }(\mathrm{C})=1\end{array}$} \\
\hline 1 & \multirow{2}{*}{$\begin{array}{c}\text { B@true } \\
\text { fst }(B)=0\end{array}$} & $\begin{array}{c}x @ \operatorname{true} \\
\text { fst }(x)=0\end{array}$ & \\
\hline 2 & & $\begin{array}{c}y @ \operatorname{true} \\
\text { fst }(y)=1\end{array}$ & \multirow{2}{*}{$\begin{array}{c}\text { C@true } \\
\text { fst }(C)=0\end{array}$} \\
\hline 3 & $\begin{array}{c}\mathrm{D} @ \text { true } \\
\text { fst }(\mathrm{D})=1\end{array}$ & & \\
\hline
\end{tabular}

(b)

Fig. 9. Example 2: At left, the result of retiming the scheduling table in Figure 8(a). At right, the result of directly applying throughput-optimizing modulo scheduling onto the specification in Figure 7.

The functional specification is also more complex, involving parallelism and different durations for the computation and communication operations. The durations of $A, B$, $C$, and $D$ on the two processors are $1,2,4$, and 1 , respectively, and transmitting over the bus any of the data produced by $A$ or $C$ takes 1 time unit.

Figure 8(a) provides the nonpipelined scheduling table produced for this example by the makespan-optimizing heuristics of Potop-Butucaru et al. [2009]. Operations $A$ and $B$ have been allocated on processor $P 1$, and operations $C$ and $D$ have been allocated on $P 2$. One communication is needed to transmit data $\mathrm{x}$ from $P 1$ to $P 2$. The makespan here is equal to the table length, which is 7 . The throughput is the inverse of the makespan (1/7).

When this scheduling table is given to our pipelining algorithm, the output is the pipelined scheduling table of Figure 8(b). The makespan remains unchanged (7), but the table length is now 5 , so the throughput is $1 / 5$. Note that the execution of operation $C$ starts in one pipelined execution cycle (at date 2), but ends in the next, at date 1 . Thus, operation $C$ has two reservations, one with $f s t(C)=0$ and one with $f s t(C)=1$.

Operations spanning over multiple execution cycles are not allowed in retimingbased techniques. Thus, if we apply retiming to the scheduling table of Figure 8(a), we obtain the pipelined scheduling table of Figure 9(a). For this example, the makespan is not changed, but the throughput is worse than the one produced by our technique $(1 / 6)$. 

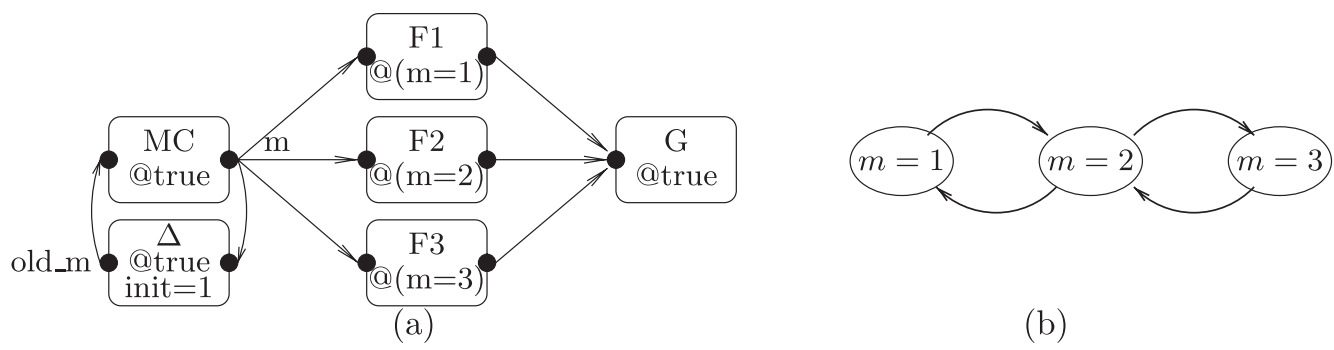

(b)

Fig. 10. Example 3: Dataflow specification with conditional execution (a) and possible mode transitions (b).

But the most interesting comparison is between the output of our pipelining technique and the result of throughput-only optimization. Figure 9(b) provides a pipelined scheduling table that has optimal throughput. In this table, operation $D$ is executed by processor $P 1$, so the bus must perform two communications. The throughput is better than in our case ( $1 / 4$ vs. $1 / 5)$, but the makespan is worse ( 8 vs. 7$)$, even though we chose a schedule with the best makespan among those with optimal throughput.

4.1.3. Example 3: Predication Handling. To explain how predication is handled in our approach, consider Figure 10. We only picture here the functional specification. As architecture, we consider two processors $P 1$ and $P 2$ connected to a shared memory $M 1$ (a two-processor version of the architecture in Figure 3(b)).

Example 3 introduces new constructs. First of all, it features a delay, labeled $\Delta$. Delays are used in our dataflow formalism to represent the system state and are a source of interiteration dependences. Each delay has an initial value, which is given as output in the first execution cycle, and then outputs at each cycle the value received as input at the previous cycle. The dataflow formalism, including delays, is formally defined in Potop-Butucaru et al. [2009].

The functional specification also makes use of conditional (predicated) execution. Operations $F 1, F 2$, and $F 3$ (of lengths 3,2 , and 1, respectively) are executed in those execution cycles where the output $m$ of operation $M C$ equals 1,2 , or 3 , respectively. Operations $M C$ and $G$ are executed in all cycles.

The output $m$ of operation $M C$ (for mode computation) is used here to represent the execution mode of our application. This mode is recomputed in the beginning of each execution cycle by $M C$, based on the previous mode and on unspecified inputs directly acquired by $M C$. We assume that the application has only three possible modes (1, 2 , and 3 ), and that transition between these modes is only possible as specified by the transitions of Figure 10(b). This constraint is specified with a predicate over the inputs and outputs of operation $M C$. All dataflow operations can be associated with such predicates, which will be used in the analyses of the next sections. Assuming that the input port of operation $M C$ is called old $m$ and that the output port is called $m$, the predicate associated to $M C$ is:

$$
\left.\left.\left(\text { old } \_m=m\right) \text { or (old_m } m=1 \text { and } m=2\right) \text { or (old } m=2\right) \text { or (old } m=3 \text { and } m=2 \text { ) }
$$

This predicate states that either there is no state change (old $\_m=m$ ), or there is a transition from state 1 to state 2 (old_ $m=1$ and $m=2$ ), or that the old state is 2 , so the new state can be any of the 3 (old_m $=2$ ), or that there is a transition from state 3 to state 2 (old_ $m=3$ and $m=2$ ).

We assume that operations $M C, F 1, F 2$, and $F 3$ are executed on processor $P 1$ and that $G$ is executed on $P 2$. Under these conditions, one possible nonpipelined schedule produced by phase 1 is the one pictured in Figure 11. Note that this table features three conditional reservations for operation $G$, even if $G$ does not have an execution 


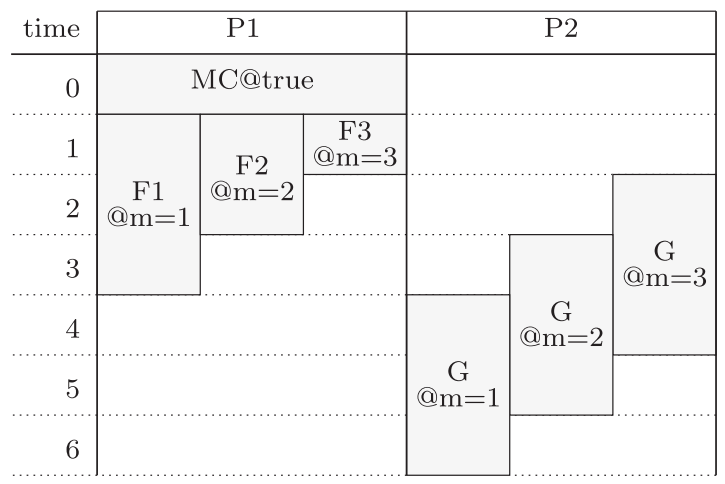

Fig. 11. Example 3: Nonpipelined scheduling table.

\begin{tabular}{|c|c|c|c|c|c|c|}
\hline time & \multicolumn{3}{|c|}{ P1 } & \multicolumn{3}{|c|}{ P2 } \\
\hline 0 & \multicolumn{3}{|c|}{ MC@true } & \multirow{3}{*}{$\begin{array}{l}@_{\mathrm{m}=1} \\
\mathrm{fst}=1\end{array}$} & \multirow{2}{*}{$\begin{array}{l}\underset{\mathrm{G}}{@=2}=2 \\
\mathrm{fst}=1\end{array}$} & \multirow[t]{2}{*}{$\begin{array}{c}\Theta_{\mathrm{m}=3}^{\mathrm{G}} \\
\mathrm{fst}=1\end{array}$} \\
\hline 1 & \multirow{3}{*}{$\begin{array}{c}\mathrm{F} 1 \\
@ \mathrm{~m}=1 \\
\mathrm{fst}=0\end{array}$} & \multirow{2}{*}{$\begin{array}{c}\mathrm{F} 2 \\
@ \mathrm{~m}=2 \\
\text { fst }=0\end{array}$} & \multirow[t]{2}{*}{$\begin{array}{l}\alpha_{\mathrm{m}=3}^{3}=3 \\
\mathrm{fst}=0\end{array}$} & & & \\
\hline 2 & & & & & & \\
\hline 3 & & & & & $\begin{array}{l}a_{\mathrm{m}}^{\mathrm{G}}=2 \\
\mathrm{fst}=0\end{array}$ & fst $=0$ \\
\hline
\end{tabular}

Fig. 12. Example 3: Pipelined scheduling table produced by our technique.

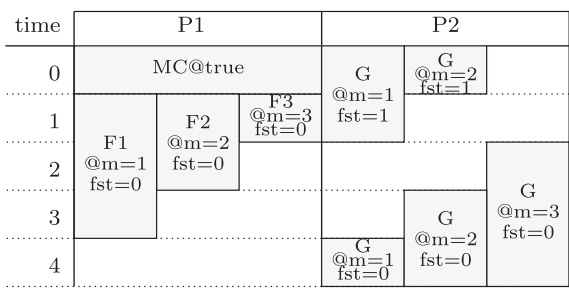

Fig. 13. Example 3: Pipelined scheduling table where intercycle execution condition analysis has not been used to improve sharing.

condition in the dataflow graph. This allows $G$ to start as early as possible in every given mode.

This table clearly features the reservation of the same resource, at the same time, by multiple operations. For instance, operations $F 1, F 2$, and $F 3$ share $P 1$ at date 1 . Of course, each time this happens, the operations must have exclusive predicates, meaning that there is no conflict at runtime.

Pipelining this table using the algorithms of the following sections produces the scheduling table of Figure 12. The most interesting aspect of this table is that the reservations $G @ m=1, f s t=1$ and $G @ m=3, f s t=0$, which belong to different execution cycles of the nonpipelined table, are allowed to overlap in time. This is possible because the dependency analysis of Section 5 determined that the two operations have exclusive execution conditions. In our case, this is due to the fact that $m$ cannot change its value directly from 1 to 3 when moving from one nonpipelined cycle to the next.

When relations between execution conditions of operations belonging to different execution cycles are not taken into account, the resulting pipelining is that of Figure 13. Here, reservations for $G$ cannot overlap in time if they have different $f s t$ values.

The current implementation of our algorithms can only analyze predicates with Boolean arguments. Thus, our three-valued mode variable $m$ needs re-encoding with two Boolean variables. In other words, in the version of Example 3 that can be processed by our tool, the operation $M C$ actually has two Boolean inputs and two Boolean outputs, and the predicate presented is defined using these four variables.

\subsection{Construction of the Pipelined Scheduling Table}

The prologues of our pipelined executions are obtained by incremental activation of the steady state operations, as specified by the $f s t$ indices (this is a classical feature 
of modulo scheduling pipelining approaches). Then, the pipelined scheduling table can be fully built using Algorithm 1 starting from the nonpipelined table and from the pipelined initiation interval. The algorithm first determines the start index and new start date of each operation by folding the nonpipelined table onto the new period. Algorithm AssembleSchedule then determines which memory cells need to be replicated due to pipelining, using the technique provided in Section 4.4.

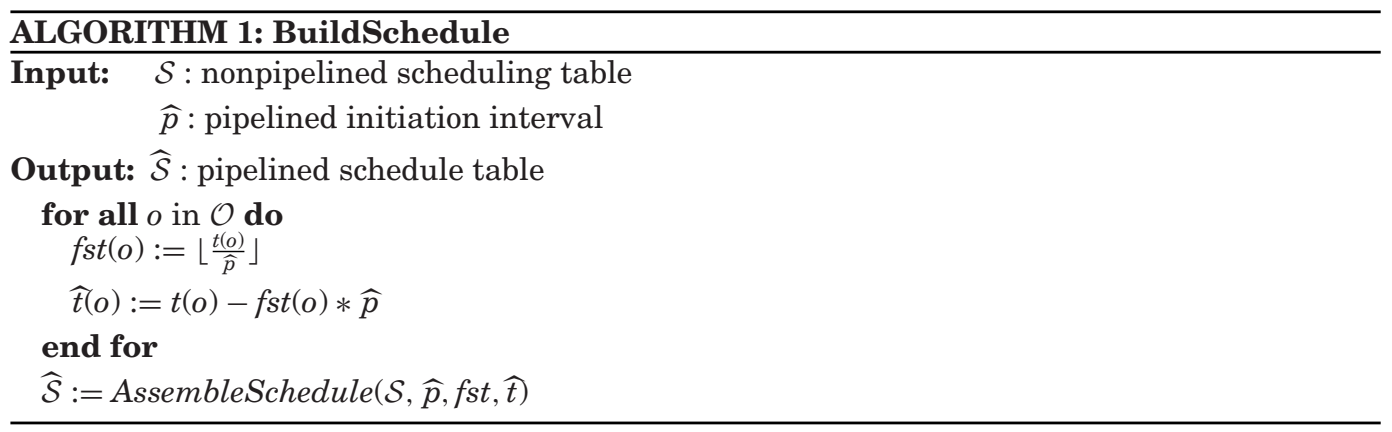

\subsection{Dependency Graph and Maximal Throughput}

The period of the pipelined system is determined by the data dependences between successive execution cycles and by the resource constraints. If we follow the classification of Hennessy and Patterson [2007], we are interested here in true data dependences and not in name dependences such as antidependences and output dependences. A true data dependency exists between two operations when one uses as input the value computed by the other. Name dependences are related to the reuse of variables and can be eliminated by variable renaming. For instance, consider the following $\mathrm{C}$ code fragment:

$$
\mathrm{x}:=\mathrm{y}+\mathrm{z} ; \mathrm{y}:=10 ; \mathrm{z}:=\mathrm{y} ;
$$

Here, there is a true data dependence (on variable y) between statements 2 and 3 . There is also an antidependence (on variable y) between statements 1 and 2 (they cannot be re-ordered without changing the result of the execution). Renaming variable y in statements 2 and 3 removes this antidependence and allows reordering of statements 1 and 2 :

$$
\mathrm{x}:=\mathrm{y}+\mathrm{z} ; \mathrm{y} 2:=10 ; \mathrm{z}:=\mathrm{y} 2 ;
$$

In our case, not needing the analysis of name dependences is due to the use of the rotating register files (detailed in Section 4.4), which remove antidependences, and to the fact that output dependences are not semantically meaningful in our systems whose execution is meant to be infinite.

We represent data dependences as a Data Dependency Graph (DDG)—a formalism that is classical in software pipelining based on modulo scheduling techniques [Allan et al. 1995]. In this section, we define DDGs and explain how the new period is computed from them. The computation of DDGs is detailed in Section 5.

Given a scheduling table $\mathcal{S}=<p, \mathcal{O}$, Init $>$, the DDG associated to $\mathcal{S}$ is a directed graph $D G=<\mathcal{O}, \mathcal{V}>$ where $\mathcal{V} \subseteq \mathcal{O} \times \mathcal{O} \times \mathbb{N}$. Ideally, $\mathcal{V}$ contains all of the triples $\left(o_{1}, o_{2}, n\right)$ such that there exists an execution of the scheduling table and a computation cycle $k$ such that operation $o_{1}$ is executed in cycle $k$, operation $o_{2}$ is executed in cycle $k+n$, and $o_{2}$ uses a value produced by $o_{1}$. In practice, any $\mathcal{V}$ including all of the arcs defined previously (any overapproximation) will be acceptable, leading to correct (but possibly suboptimal) pipelinings. 
The DDG represents all possible dependences between operations, both inside a computation cycle (when $n=0$ ) and between successive cycles at distance $n \geq 1$. Given the static scheduling approach, with fixed dates for each operation, the pipelined scheduling table must respect unconditionally all of these dependences.

For each operation $o \in \mathcal{O}$, we denote with $t_{n}(o)$ the date where operation $o$ is executed in cycle $n$, if its guard is true. By construction, we have $t_{n}(o)=t(o)+n * p$. In the pipelined scheduling table of period $\widehat{p}$, this date is changed to $\widehat{t}_{n}(o)=t(o)+n * \widehat{p}$. Then, for all $\left(o_{1}, o_{2}, n\right) \in \mathcal{V}$ and $k \geq 0$, the pipelined scheduling table must satisfy $\widehat{t_{k+n}}\left(o_{2}\right) \geq \widehat{t_{k}}\left(o_{1}\right)+d\left(o_{1}\right)$, which implies:

$$
\widehat{p} \geq \max _{\left(o_{1}, o_{2}, n\right) \in \mathcal{V}, n \neq 0}\left\lceil\frac{t\left(o_{1}\right)+d\left(o_{1}\right)-t\left(o_{2}\right)}{n}\right\rceil
$$

Our objective is to build pipelined scheduling tables satisfying this lower bound constraint and that are well formed in the sense of Section 3.3.

\subsection{Memory Management Issues}

Our pipelining technique allows multiple instances of a given variable, belonging to successive nonpipelined execution cycles, to be simultaneously live. For instance, in Figures 4 and 5 , both $A$ and $B$ use memory cell $v_{1}$ at each cycle. In the pipelined table, $A$ and $B$ work in parallel, so they must use two different copies of $v_{1}$. In other words, we must provide an implementation of the expanded virtual registers of Rau [1996].

The traditional software solution to this problem is the modulo variable expansion of Lam [1988]. However, this solution requires loop unrolling, which would increase the size of our scheduling tables. Instead, we rely on a purely software implementation of rotating register files [Rau et al. 1992], which requires no loop unrolling nor special hardware support. Our implementation of rotating register files includes an extension for identifying the good register to read in the presence of predication. To our best knowledge, this extension (detailed in Section 4.4.2) is an original contribution.

4.4.1. Rotating Register Files for Stateless Systems. Assuming that $\widehat{\mathcal{S}}$ is the pipelined version of $\mathcal{S}$, we denote with $\max _{-} \operatorname{par}=\lceil\operatorname{len}(\mathcal{S}) / \operatorname{len}(\widehat{\mathcal{S}})\rceil$ the maximal number of simultaneously active computation cycles of the pipelined scheduling table. Note that $\max _{-}$par $=1+\max _{o \in \mathcal{O}} f s t(o)$.

In Figures 4 and 5 , we must use two different copies of $v_{1}$. We will say that the replication factor of $v_{1}$ is $\operatorname{rep}\left(v_{1}\right)=2$. Each memory cell $v$ is assigned its own replication factor, which must allow concurrent nonpipelined execution cycles using different copies of $v$ to work without interference. Obviously, we can bound rep $(v)$ by max_par. We use a tighter margin, based on the observation that most variables (memory cells) have a limited lifetime inside a nonpipelined execution cycle. We set $\operatorname{rep}(v)=1+\operatorname{lst}(v)-f s t(v)$, where:

$$
f s t(v)=\min _{v \in \operatorname{In}(o) \cup O u t(o)} f s t(o) \quad l s t(v)=\max _{v \in \operatorname{In}(o) \cup O u t(o)} f s t(o)
$$

Through replication, each memory cell $v$ of the nonpipelined scheduling table is replaced by $\operatorname{rep}(v)$ memory cells, allocated on the same memory block as $v$, and organized in an array $\bar{v}$, whose elements are $\bar{v}[0], \ldots, \bar{v}[\operatorname{rep}(v)-1]$. These new memory cells are allocated cyclically, in a static fashion, to the successive nonpipelined cycles. More precisely, the nonpipelined cycle of index $n$ is assigned the replicas $\bar{v}[n \bmod \operatorname{rep}(v)]$ for all $v$. The computation of $\operatorname{rep}(v)$ ensures that if $n_{1}$ and $n_{2}$ are equal modulo $\operatorname{rep}(v)$, but $n_{1} \neq n_{2}$, then computation cycles $n_{1}$ and $n_{2}$ cannot access $v$ at the same time.

To exploit replication, the code generation scheme must be modified by replacing $v$ with $\bar{v}[(c i d-f s t(o)) \bmod \operatorname{rep}(v)]$ in the input and output parameter lists of every operation $o$ that uses $v$. Here, cid is the index of the current pipelined cycle. It is 
represented in the generated code by an integer. When execution starts, cid is initialized with 0 . At the start of each subsequent pipelined cycle, it is updated to $(c i d+1) \bmod R$, where $R$ is the least common multiple of all values $\operatorname{rep}(v)$.

This simple implementation of rotating register files allows code generation for systems where no information is passed from one nonpipelined execution cycle to the next (no intercycle dependences). Such systems, such as the example in Figures 4 and 5, are also called stateless.

4.4.2. Extension to Stateful Predicated Systems. In stateful systems, one (nonpipelined) execution cycle may use values produced in previous execution cycles. In these cases, code generation is more complicated, because an execution cycle must access memory cells that are not its own.

For certain classes of applications (such as systems without conditional control or affine loop nests), the cells to access can be statically identified by offsets with respect to the current execution cycle. For instance, the $M C$ mode change function of Example 3 (Section 4.1.3) always reads the variable $m$ produced in the previous execution cycle.

But in the general case, in the presence of predicated execution, it is impossible to statically determine which cell to read, as the value may have been produced at an arbitrary distance in the past. This is the case if, for instance, the data production operation is itself predicated. One solution to this problem is to allow the copying of one memory cell onto another in the beginning of pipelined cycles. But we cannot accept this solution due to the nature of our data, which can be large tables for which copying implies large timing penalties.

Instead, we modify the rotating register file as follows: storage is still ensured by the $\bar{v}$ circular buffer, which has the same length. However, its elements are not directly addressed through the modulo counter (cid - fst $(v))$ mod rep $(v)$ used earlier. Instead, this counter points in an array $\operatorname{src}_{v}$ whose cells are integer indices pointing toward cells of $\bar{v}$. This allows operations from several nonpipelined execution cycles (with different cid and $f s t(o))$ to read the same cell of $\bar{v}$, eliminating the need for copying.

The full implementation of our register file requires two more variables: an integer next $t_{v}$ and an array of Booleans write flag $v$ of length rep $(v)$. Since $\bar{v}$ is no longer directly addressed through the modulo counter, next $t_{v}$ is needed to implement the circular buffer policy of $\bar{v}$ by pointing to the cell where a newly produced value can be stored next. One cell of $\bar{v}$ is allocated (and next $t_{v}$ incremented) whenever a nonpipelined execution cycle writes $v$ for the first time. Subsequent writes of $v$ from the same nonpipelined cycle use the already allocated cell. Determining whether a write is the first from a given nonpipelined execution cycle is realized using the flags of write flag ${ }_{v}$. Note that the use of these flags is not needed when a variable can be written at most once per execution cycle. This is often the case for code used in embedded systems design, such as the output of the Scade language compiler, or the output of the makespan-optimizing scheduling tool used for evaluation. If a given execution cycle does not write $v$, then it must read the same memory cell of next ${ }_{v}$ that was used by the previous execution cycle.

The resulting code generation scheme is precisely described by the following rules:

(1) At application start, for every every memory cell (variable) $v$ of the initial specification, $\operatorname{src}_{v}[0]$, write flag $_{v}[0]$, next $_{v}$ are initialized, respectively, with 0 , false, and 1 . If $\operatorname{Init}(v) \neq n i l$, then $\bar{v}[0]$ (instead of $v$ ) is initialized with this value.

(2) At the start of each pipelined cycle, for memory cell (variable) $v$ of the initial scheduling table, assign to $\operatorname{src}_{v}[(\operatorname{cid}-f s t(v)) \bmod \operatorname{rep}(v)]$ the value of $\operatorname{src}_{v}\left[\left(c^{2} i d-\right.\right.$ $f s t(v)-1) \bmod r e p(v)]$, and set write_flag $_{v}[(\operatorname{cid}-f s t(v)) \bmod \operatorname{rep}(v)]$ to false.

(3) In all operations $o$ replace each input and output cell $v$ with $\bar{v}\left[\operatorname{src}_{v}[(\operatorname{cid}-f s t(o)) \bmod \right.$ $\operatorname{rep}(v)]]$. The same must be done for all cells used in the computation of execution conditions. 
(4) When an operation $o$ has $v$ as an output parameter, then some code must be added before the operation (inside its execution condition). There are two cases. If $v$ is not an input parameter of $o$, then the code is the following:

1: if not write flag $_{v}[(\operatorname{cid}-f s t(o)) \bmod \operatorname{rep}(v)]$ then

2: write flag $_{v}\left[\left(\mathrm{cid}-\mathrm{fst}_{(\mathrm{o}))} \bmod \mathrm{rep}(\mathrm{v})\right]=\right.$ true

3: $\operatorname{src}_{v}[(\operatorname{cid}-f s t(o)) \bmod \operatorname{rep}(v)]=\operatorname{next}_{v}$

4: next $_{v}=\left(\right.$ next $\left._{v}+1\right) \bmod \operatorname{rep}(v)$

5: end if

If $v$ is also an input parameter of $o$, then only line 2 is needed from the previous code.

4.4.3. Accounting for Bookkeeping Costs. The software implementation of the rotating register files induces a timing overhead of its own. This overhead is formed of two components:

- Per operation costs, which can be conservatively accounted for in the WCET of the operations, as it is provided to phase 1 of our scheduling flow:

-For each operation and for each input and output parameter, the cost of the indirection of point (3). This amounts to two indirections, one addition, and one modulo operation.

-For each operation and for each output parameter, the cost of the bookkeeping operations defined at point (4).

-Per iteration costs, associated with point (2) and that must be added to the length of the pipelined scheduling table. This amounts to updating the $\operatorname{src}_{v}$ and write flag ${ }_{v}$ data structures for all $v$. These costs are also bounded and can be accounted for with worst-case figures in phase 1.

One important remark here is that our operations are large-grain tasks, meaning that these costs are often considered negligible, even for hard real-time applications.

The use of rotating registers also results in memory usage overheads. These overheads come from the replication of memory cells and from the pointer arrays $\operatorname{src}_{v}$, which must be stored on the same memory bank as $v$ for all memory cells $v$. We will assume that the cost of src is negligible and will only be concerned with the cost of replication, especially for large data.

If the replication of a large piece of data is a concern, then we can prohibit it altogether by requiring that all accesses to that memory cell are sequenced. This is done by adding a "sequencer" processor to the architecture model and requiring all accesses to that memory cell to use the sequencer processor (this requires modifications to both the architecture and the functional specification). The introduction of sequencers may limit the efficiency of our pipelining algorithms. However, being able to target specific memory cells means that we can limit efficiency loss to what is really necessary on our memory-constrained embedded platforms. This simple approach satisfies our current needs.

\section{DEPENDENCY ANALYSIS AND MAIN ROUTINE}

Dependency analysis is a mature discipline, and powerful algorithms have been used in practice for decades [Muchnick 1997]. However, previous research on interiteration dependencyanalysis has mostly focused on exploiting the regularity of code such as affine loop nests. To the best of our knowledge, existing algorithms are unable to analyze specifications such as our Example 3 (Section 4.1.3) with the precision that we seek. Doing this requires that interiteration dependency analysis deals with datadependent mode changes (which are a common feature in embedded systems design). 


\begin{tabular}{r|c|c|c|}
\cline { 2 - 4 } time & P1 & P2 & P3 \\
\hline 0 & A@true & & \\
\cline { 2 - 4 } 1 & & B@true & \\
\cline { 3 - 4 } 2 & & & C@true \\
\cline { 2 - 3 } & & & \\
\cline { 2 - 3 } & & &
\end{tabular}

Fig. 14. Dependency analysis example, nonpipelined.

\begin{tabular}{r|c|c|c|}
\cline { 2 - 4 } time & P1 & P2 & P3 \\
\hline \multirow{2}{*}{0} & $\begin{array}{c}\mathrm{A} @ \text { true } \\
f s t(A)=0\end{array}$ & & $\begin{array}{c}\text { C@true } \\
f s t(C)=1\end{array}$ \\
\cline { 2 - 4 } 1 & $\begin{array}{c}\mathrm{D} @ \text { true } \\
f s t(D)=1\end{array}$ & $\begin{array}{c}\mathrm{B} @ \text { true } \\
f s t(B)=0\end{array}$ &
\end{tabular}

Fig. 15. Dependency analysis example, pipelined.

Performing our precise interiteration dependency analysis requires the (potentially infinite) unrolling of the nonpipelined scheduling table. But our specific pipelining technique allows us to bound the unrolling and thus limit the complexity of dependency analysis. By comparison, existing pipelining and PAS techniques either assume that the dependency graph is fully generated before starting the pipelining algorithm [Rau and Glaeser 1981] or use the predicates for the analysis of a single cycle [Warter et al. 1993].

The core of our dependency analysis consists in lines 1 through 10 of Algorithm 3, which act as a driver for Algorithm 2. The remainder of Algorithm 3 uses DDG-derived information to drive the pipelining routine (Algorithm 1).

Both the data dependency analysis and pipelining driver take as input a flag that chooses between two pipelining modes with different complexities and capabilities. To understand the difference, consider the nonpipelined scheduling table of Figure 14. Resource $P_{1}$ has an idle period between operations $A$ and $D$ where a new instance of $A$ can be started. However, to preserve a periodic execution model, $A$ should not be restarted just after its first instance (at date 1). Indeed, this would imply a pipelined throughput of 1 , but the fourth instance of $A$ cannot be started at date 3 (only at date 6 ). The correct pipelining starts $A$ at date 2 and results in the pipelined scheduling table of Figure 15.

Determining if the reuse of idle spaces between operations is possible consists in determining the smallest integer $n$ greater than the lower bound of Section 4.3, smaller than the length of the initial table, and such that a well-formed pipelined table of length $n$ can be constructed. This computation is performed by lines 14 through 19 of Algorithm 3. We do not provide here the code of function WellFormed, which checks the respect of the well-formed properties of Section 3.3.

This complex computation can be avoided when idle spaces between two operations are excluded from use at pipelining time. This can be done by creating a dependency between any two operations of successive cycles that use a same resource and have nonexclusive execution conditions. In this case, the pipelined system period is exactly the lower bound of Section 4.3, and the output scheduling table is produced with a single call to Algorithm 1 (BuildSchedule) in line 12 of Algorithm 3. Of course, Algorithm 2 needs to consider (in lines 10 through 16) the extra dependences.

Excluding the idle spaces from pipelining also has the advantage of supporting a sporadic execution model. In sporadic systems, the successive computation cycles can be executed with the maximal throughput specified by the pipelined table but can also be triggered arbitrarily less often-for instance, to tolerate timing variations or to minimize power consumption in systems where the demand for computing power varies. On the contrary, using the idle spaces during pipelining imposes synchronization constraints between successive execution cycles. For instance, in the pipelined system of Figure 15, the computation cycle of index $n$ cannot complete before operation $A$ of cycle $n+1$ is completed. 


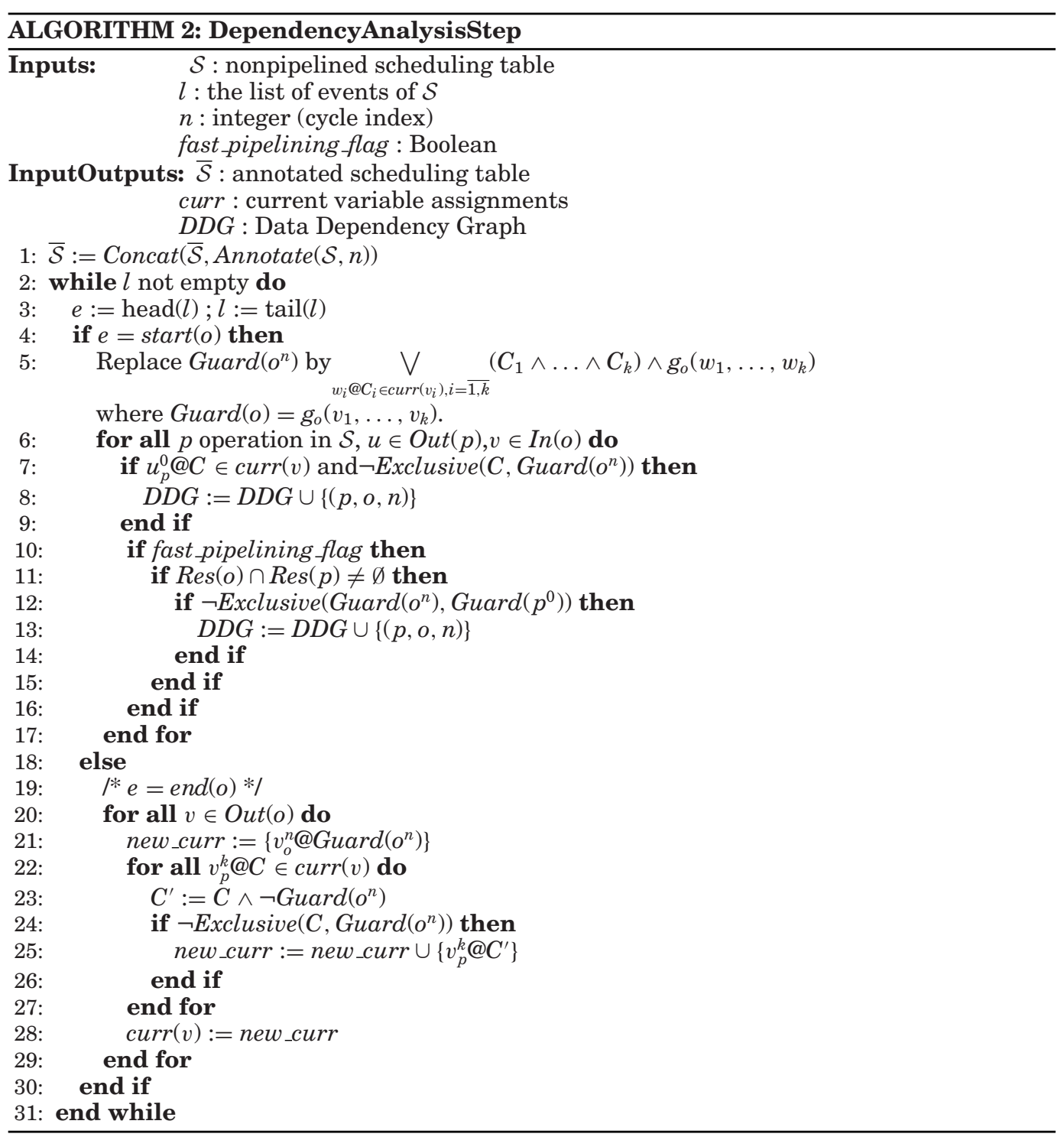

The remainder of this section details the dependency analysis phase. The output of this analysis is the lower bound defined in Section 4.3, computed as period_lbound. The analysis is organized around the repeat loop that incrementally computes, for cycle $\geq 1$, the DDG dependences of the type $\left(o_{1}, o_{2}\right.$, cycle). The computation of the DDG is not complete: we bound it using a loop termination condition derived from our knowledge of the pipelining algorithm. This condition is based on the observation that if period_lbound $* k \geq \operatorname{len}(\mathcal{S})$, then execution cycles $n$ and $n+k$ cannot overlap in time (for all $n$ ).

The DDG computation works by incrementally unrolling the nonpipelined scheduling table. At each unrolling step, the result is put in the $\mathrm{SSA}^{3}$-like data structure $\overline{\mathcal{S}}$ that

\footnotetext{
${ }^{3}$ SSA stands for Static Single Assignment representation form [Muchnick 1997].
} 
allows the computation of (an overapproximation of) the dependency set. Unrolling is done by annotating each instance of an operation $o$ with the cycle $n$ in which it has been instantiated. The notation is $o^{n}$. Putting in SSA-like form is based on splitting each memory cell $v$ into one version per operation instance producing it $\left(v_{o}^{n}\right.$, if $v \in$ Out $\left.(o)\right)$ and one version for the initial value $\left(v_{\text {init }}\right)$. Annotation and variable splitting is done on a per-cycle basis by the Annotate routine (not provided here), which changes for each operation $o$ its name to $o^{n}$ and replaces Out(o) with $\left\{v_{o}^{n} \mid v \in \operatorname{Out}(o)\right\}$ (here, $n$ is the cycle index parameter). Instances of $\mathcal{S}$ produced by Annotate are then assembled into $\overline{\mathcal{S}}$ by the Concat function, which simply adds to the date of every operation in the second argument the length of its first argument.

Recall that we are only interested in dependences between operations in different cycles. Then, in each call to Algorithm 2, we determine the dependences between operations ofcycle 0 and operations of cycle $n$, where $n$ is the current cycle. To determine them, we rely on a symbolic execution of the newly added part of $\overline{\mathcal{S}}$ - that is, the operations $o^{k}$ with $k=n$. Symbolic execution is done through a traversal of list $l$, which contains all operation start and end events of $\mathcal{S}$, and therefore $\overline{\mathcal{S}}$, ordered by increasing date. For each operation $o$ of $\mathcal{S}, l$ contains two elements labeled $\operatorname{start}(o)$ and end $(o)$. The list is ordered by increasing the event date using the convention that the date of $\operatorname{start}(o)$ is $t(o)$ and the date of end $(o)$ is $t(o)+d(o)$. Moreover, if $\operatorname{start}(o)$ and $\operatorname{end}\left(o^{\prime}\right)$ have the same date, the start(o) event comes first in the list.

At each point of the symbolic execution, the data structure curr identifies the possible producers of each memory cell. For each cell $v$ of the initial table, $\operatorname{curr}(v)$ is a set of pairs $w @ C$, where $w$ is a version of $v$ of the form $v_{o}^{k}$ or $v_{\text {init }}$, and $C$ is a predicate over memory cell versions. In the pair $w @ C, C$ gives the condition on which the value of $v$ is the one corresponding to its version $w$ at the considered point in the symbolic simulation. Intuitively, if $v_{o}^{k} C \in \operatorname{curr}(v)$, and we symbolically execute cycle $n$, then $C$ gives the condition under which in any real execution of the system $v$ holds the value produced by $o, n-k$ cycles before. The predicates of the elements in $\operatorname{curr}(v)$ provide a partition of true. Initially, $\operatorname{curr}(v)$ is set to $\left\{v_{\text {init }} @\right.$ true $\}$ for all v. This is changed by Algorithm 2 (lines 20 through 29) and by the call to InitCurr in Algorithm 3. We do not provide this last function, which performs the symbolic execution of the nodes of $\overline{\mathcal{S}}$ annotated with 0 . Its code is virtually identical to that of Algorithm 2, lines 1 and 6 through 17 being excluded.

At each operation start step of the symbolic execution, curr allows us to complete the SSA transformation by recomputing the guard of the current operation over the split variables (line 5 of Algorithm 2). In turn, this allows the computation of the dependences (lines 6 through 17). Guard comparisons are translated into predicates that are analyzed by a SAT solver. This translation into predicates also considers the predicates relating inputs and outputs of the dataflow operations, as intuitively explained in Section 4.1.3. The translation and the callto SAT are realized by the Exclusive function, not provided here.

\subsection{Complexity Considerations}

The pipelining algorithm per se consists in Algorithm 1 and its driver (lines 11 through 20 of Algorithm 3). The complexity of Algorithm 1 is linear in the number of operations in the scheduling table. As explained earlier, the complexity of the driver routine (lines 11 through 20 of Algorithm 3) depends on the value of fast_pipelining_flag. When it is set to true, a single call to Algorithm 1 is performed. When it is set to false, the number of calls to Algorithm 1 is bounded by len $(\mathcal{S})$.

In our experiments, fast_pipelining_flag is set for all examples, and the pipelining time is negligible. 


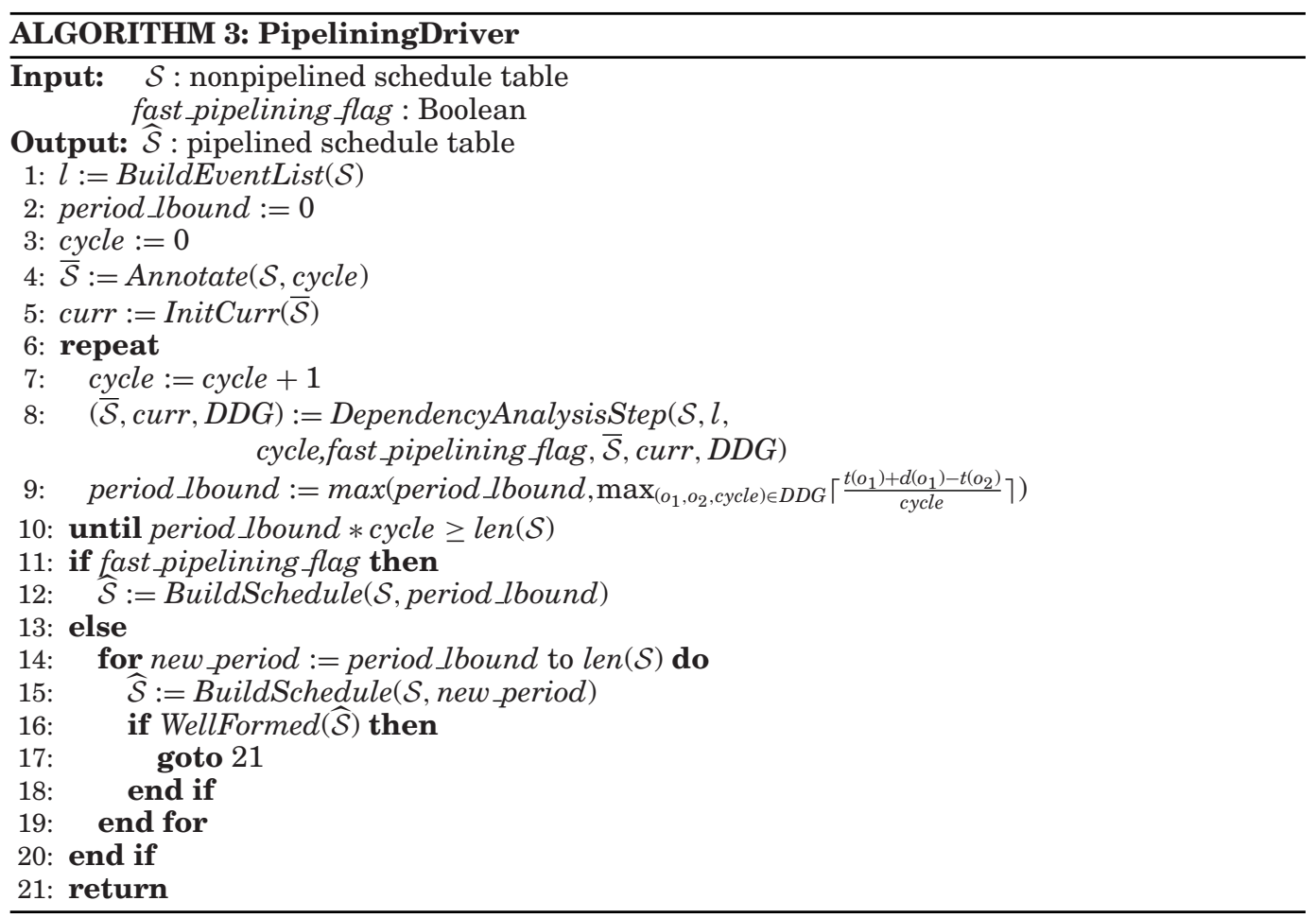

But the main source of theoretical complexity in our pipelining technique is hidden in the dependency analysis implemented by Algorithm 2 and its driver (lines 1 through 10 or Algorithm 3). In Algorithm 3, the number of iterations in the construction of the DDG is upbounded by len $(\mathcal{S}$ ) (which can be large). Algorithm 2 is polynomial (quadratic) in the number of operations of the scheduling table but involves comparisons of predicates with Boolean arguments (instances of the Boolean satisfiability problem SAT). Hence, our algorithm is overall NP-complete.

In practice, however, DDG construction time is negligible for all examples, real-life and synthesized. There are two reasons for this:

-In examples featuring predicated execution, the predicates remain simple, so SAT instances are solved in negligible time.

- The number of iterations in the construction of the DDG is bounded by the condition in line 10 of Algorithm 3. In our experiments, the maximal number of iterations is 2 .

\section{EXPERIMENTAL RESULTS}

We have implemented our pipelining algorithms in a prototype tool. We have integrated this tool with an existing makespan-optimizing scheduling tool [Potop-Butucaru et al. 2009] to form the full, two-phase flow of Figure 1.

We have applied the resulting toolchain on four significant, real-life examples from the testbench of the SynDEx scheduling tool [Grandpierre and Sorel 2003]. As no standard benchmarks are available in the embedded world, we have also applied our toolchain on a larger number of automatically synthesized dataflow graphs. 


\begin{tabular}{c|c|c|c|c|c}
\multirow{2}{*}{ example } & \multicolumn{2}{|c|}{ Example size } & \multicolumn{3}{c}{$\begin{array}{c}\text { Scheduling table length } \\
\text { (initiation interval) }\end{array}$} \\
\cline { 2 - 6 } & blocks & processors & $\begin{array}{c}\text { initial } \\
\text { (makespan) }\end{array}$ & $\begin{array}{c}\text { pipelined } \\
\text { (kernel) }\end{array}$ & gain \\
\hline cycab & 40 & 3 & 1482 & 1083 & $27 \%$ \\
robucar & 84 & 3 & 1093 & 1053 & $8 \%$ \\
ega & 67 & 2 & 84 & 79 & $6 \%$ \\
knock & 5 & 2 & 6 & 3 & $50 \%$
\end{tabular}

Fig. 16. Pipelining gains for the real-life applications. Durations are in time units whose actual real-time length depends on the application (e.g., milliseconds).

Our objective was to evaluate both the standalone pipelining algorithm and the twophase flow as a whole. Comparing with optimal scheduling results was not possible. ${ }^{4}$ Instead, we rely on comparisons with existing scheduling and pipelining heuristics:

(1) To evaluate the standalone algorithm, we measure the throughput gains obtained through pipelining, by comparing the initiation intervals of our examples before and after pipelining.

(2) To evaluate the two-phase flow as a whole, we compare its output to the output of a classical throughput-optimizing software pipelining technique, namely the FRLC algorithm of Wang and Eisenbeis [1993].

The testbench. The largest examples of our testbench ("cycab" and "robucar") are embedded control applications for the CyCab electric car [Pradalier et al. 2005]. The other two applications are an adaptive equalizer and a simplified model of an automotive knock control application [André et al. 2007].

We have used a script to automatically synthesize 30 examples (of which the first 10 are also presented individually in the result tables). For each example, synthesis is done as follows. We start with a graph containing only one dataflow node and no dependency. We apply a fixed number of expansion steps (three steps for the examples in Figure 17). At every step, each node is replaced with either a parallel or a sequential composition of newly created nodes. The sequential and parallel choices are equiprobable. The number of nodes generated through expansion is chosen with a uniform distribution in the interval [1..5]. Dependences are also generated randomly at each step, and all previously existing dependences are preserved. We implement these dataflow graphs on an architecture containing five processors and one broadcast bus. To model the fact that the architecture is not homogenous, the durations of the dataflow blocks on the various processors are assigned randomly (uniform distribution in an interval), and we randomly create a small number of placement constraints. We also assume that one of the processors performs input acquisition and another processor controls actuators. This implies placement constraints on dataflow blocks with no inputs and no outputs, respectively.

Pipelining gains. The pipelining gains for the real-life and synthesized examples are summarized in Figures 16 and 17, respectively. The figures show improvements on all examples, with a reduction of $27 \%$ in cycle time for the large "cycab" example and an average reduction of $9.31 \%$ on the synthesized examples. We conclude that a pipelining stage such as ours should be part of any static scheduling flow.

At the same time, improvement varies greatly among the examples, from $50 \%$ for the knock control to $4 \%$ for one of the generated examples. We inspected the examples

${ }^{4}$ Providing optimal solutions to our scheduling problem proved intractable even for small systems with 10 blocks and three processors. 


\begin{tabular}{l|c|c|c|c|c}
\multirow{2}{*}{ Example } & \multicolumn{2}{|c|}{ Example size } & \multicolumn{3}{c}{$\begin{array}{c}\text { Scheduling table length } \\
\text { (initiation interval) }\end{array}$} \\
\cline { 2 - 6 } & blocks & processors & $\begin{array}{c}\text { initial } \\
\text { (makespan) }\end{array}$ & $\begin{array}{c}\text { pipelined } \\
\text { (kernel) }\end{array}$ & gain \\
\hline synth1 & 34 & 5 & 463 & 430 & $7 \%$ \\
synth2 & 35 & 5 & 315 & 290 & $7.9 \%$ \\
synth3 & 11 & 5 & 171 & 142 & $16.9 \%$ \\
synth4 & 52 & 5 & 840 & 793 & $5 \%$ \\
synth5 & 23 & 5 & 375 & 348 & $7 \%$ \\
synth6 & 46 & 5 & 433 & 310 & $28 \%$ \\
synth7 & 14 & 5 & 212 & 191 & $9.9 \%$ \\
synth8 & 40 & 5 & 622 & 594 & $4 \%$ \\
synth9 & 15 & 5 & 351 & 320 & $8.8 \%$ \\
synth10 & 29 & 5 & 318 & 291 & $8 \%$ \\
\hline Average for 30 examples
\end{tabular}

Fig. 17. Pipelining gains for the synthesized examples.

\begin{tabular}{l|c|c|c|c|c|c} 
& \multicolumn{2}{|c|}{ Our method } & [Wang and Eisenbeis 1993] & Makespan & Throughput \\
Example & makespan & kernel & makespan & kernel & gain & loss \\
\hline synth1m & 298 & 276 & 850 & 246 & $64 \%$ & $12 \%$ \\
synth2m & 277 & 263 & 570 & 190 & $51 \%$ & $38 \%$ \\
synth3m & 142 & 142 & 299 & 142 & $52 \%$ & $0 \%$ \\
synth4m & 347 & 328 & 2380 & 255 & $85 \%$ & $28 \%$ \\
synth5m & 155 & 155 & 651 & 155 & $76 \%$ & $0 \%$ \\
synth6m & 349 & 288 & 1774 & 288 & $80 \%$ & $0 \%$ \\
synth7m & 130 & 115 & 258 & 115 & $49 \%$ & $0 \%$ \\
synth8m & 376 & 357 & 610 & 290 & $38 \%$ & $23 \%$ \\
synth9m & 116 & 96 & 198 & 82 & $41 \%$ & $17 \%$ \\
synth10m & 300 & 273 & 1686 & 96 & $82 \%$ & $184 \%$ \\
\hline Average for 30 examples
\end{tabular}

Fig. 18. Comparison with a classical software pipelining algorithm.

showing poor performance. Some of them, like "ega" have very tight schedules with little idle CPU time and therefore little opportunity for pipelining. More interesting was "robucar," which has significant idle time but where a critical path in the scheduling table blocks pipelining. For such cases, more powerful pipelining algorithms are needed (as part of future work), able to modify the scheduling of the nonpipelined execution cycles but without lengthening the makespan.

Comparison with a classical software pipelining algorithm. To make this comparison, we have implemented the classical FRLC algorithm of Wang and Eisenbeis [1993]. We chose this algorithm because of its flexibility. It is easy to extend it to cover aspects taken into account by our tool, such as the presence of operations that have different durations on different functional units, or communication costs. However, we have used here for comparisons its baseline, restricted version. We therefore considered only the synthesized examples, which have a simpler structure, and modified them by removing communication costs and by choosing a single duration for each operation on all processors that can execute it.

On these modified examples, we applied our two-phase flow and the FRLC method and compared the results in Figure 18. For each example and scheduling flow, we provide the makespan and the kernel length of the generated code. We have also 
computed the makespan gain and throughput loss when moving from the FRLC technique to ours.

In these figures, the makespan-throughput trade-off is clearly visible (as we expected). On average, our method gains $63.63 \%$ in makespan while losing $38.33 \%$ in throughput. A less expected result is that this trade-off can be identified in each example, even though the algorithms under comparison are heuristics. Indeed, our method is always better inmakespan, whereas the FRLC technique of Wang and Eisenbeis [1993] is never worse in throughput.

Given our optimization objective (makespan first, throughput second), we consider that our choice of two-phase optimization heuristic is justified.

Predication is present in three of our examples: Example 3, knock, and a variant of the cycab example. In all of these examples, predication is used to encode modedependent behavior, and the examples showcase different situations where pipelining is necessary in embedded systems design. Mode-dependent behavior is usually specified at the level of full systems or large subsystems, meaning that the number of predicates is usually low, yet each predicate controls a significant part of the operations of the system. Our examples have only two or three predicates (two in knock and cycab, three in Example 3). To evaluate the contribution of predication analysis to the pipelining results, we have also run our algorithms with the predication analysis disabled. The use of predication does not improve pipelining result for the cycab example. On the contrary, it results in significant gains for Example 3 and knock (20\% and 40\%, respectively).

\section{CONCLUSION}

We have defined a new software pipelining technique adapted for the implementation of hard real-time multiprocessor embedded control systems. Our technique reuses the basic concepts of both modulo scheduling and decomposed software pipelining. At the same time, our technique has two significant originality points, determined by the needs of our real-time implementation problems:

-It is driven by a makespan optimization objective, with throughput as a secondary objective.

-It takes into account relations between (data-dependent) execution conditions of operations, including those for operations belonging to different execution cycles.

These two points also require revisiting classical results on rotating register files and data dependence analysis.

We have implemented our pipelining technique into a tool, and we evaluated our technique, with good results, on several real-life systems and generated scheduling problems.

The resulting flow satisfies the implementation needs for certain classes of embedded systems. But in other cases (mentioned in Section 1), separating the implementation process in two phases (scheduling followed by pipelining) is not a good idea. For such cases, we are now developing single-phase scheduling techniques. Initial results in this direction aredocumented elsewhere [Carle et al. 2012].

\section{ACKNOWLEDGMENTS}

The insightful comments of Albert Cohen on early versions of this article have allowed us to significantly improve our work.

\section{REFERENCES}

V. H. Allan, R. B. Jones, R. M. Lee, and S. J. Allan. 1995. Software pipelining. ACM Computing Surveys 27, $3,367-432$. 
C. André, F. Mallet, and M.-A. Peraldi-Frati. 2007. A multiform time approach to real-time system modeling: Application to an automotive system. In Proceedings of the SIES. Lisbon, Portugal.

ARINC653. 2005. Avionics Application Software Standard Interface, volumes 1-3. http://www.arinc.org.

AUTOSAR. 2009. Automotive Open System Architecture, release 4. http://www.autosar.org/.

A. Benoît, V. Rehn-Sonigo, and Y. Robert. 2007. Multi-criteria scheduling of pipeline workflows. In Proceedings of the International Conference on Cluster Computing. Austin, TX.

P.-Y. Calland, A. Darte, and Y. Robert. 1998. Circuit retiming applied to decomposed software pipelining. IEEE Transactions on Parallel and Distributed Systems 9, 1, 24-35.

T. Carle, D. Potop-Butucaru, Y. Sorel, and D. Lesens. 2012. From dataflow specification to multiprocessor partitioned time-triggered real-time implementation. Technical Report. INRIA. http://hal.inria.fr/ hal-00742908

P. Caspi, A. Curic, A. Magnan, C. Sofronis, S. Tripakis, and P. Niebert. 2003. From Simulink to SCADE/Lustre to TTA: A layered approach for distributed embedded applications. In Proceedings of the LCTES. San Diego, CA.

K. Chatha and R. Vemuri. 2002. Hardware-software partitioning and pipelined scheduling of transformative applications. IEEE Transactions on Very Large Scale Integration (VLSI) Systems 10, 3, 193-208.

Y.-S. Chiu, C.-S. Shih, and S.-H. Hung. 2011. Pipeline schedule synthesis for real-time streaming tasks with inter/intra-instance precedence constraints. In Proceedings of DATE. Grenoble, France.

P. Eles, A. Doboli, P. Pop, and Z. Peng. 2000. Scheduling with bus access optimization for distributed embedded systems. IEEE Transactions on VLSI Systems 8, 5, 472-491.

G. Fohler, A. Neundorf, K.-E. Årzén, C. Lucarz, M. Mattavelli, V. Noel, C. von Platen, G. Butazzo, E. Bini, and C. Scordino. 2008. EU FP7 ACTORS project. Ch 5: Resource reservation in real-time systems. In D7a: State of the Art Assessment. http://www3.control.lth.se/user/karlerik/Actors/d7a-rev.pdf.

M. R. Garey and D. S. Johnson. 1979. Computers and Intractability: A Guide to the Theory of NPCompleteness. W. H. Freeman and Company.

F. Gasperoni and U. Schwiegelshohn. 1994. Generating close to optimum loop schedules on parallel processors. Parallel Processing Letters 4, 4, 391-404.

R. Govindarajan, E. Altman, and G. Gao. 1994. Minimizing register requirements under resource-constrained rate-optimal software pipelining. In Proceedings of the 27th Annual International Symposium on Microarchitecture (MICRO 27).

T. Grandpierre and Y. Sorel. 2003. From algorithm and architecture specification to automatic generation of distributed real-time executives. In Proceedings of the MEMOCODE. Mont St. Michel, France.

J. L. Hennessy and D. A. Patterson. 2007. Computer Architecture: A Quantitative Approach (4th ed.). Morgan Kaufmann.

R. Huff. 1993. Lifetime-sensitive modulo scheduling. In Proceedings of the ACM SIGPLAN 93rd Conference on Programming Language Design and Implementation. 258-267.

W. Kim, D. Yoo, H. Park, and M. Ahn. 2012. SCC based modulo scheduling for coarse-grained reconfigurable processors. In Proceedings of the 2012 International Converence on Field-Programmable Technology (FPT). Seoul, Korea.

M. Lam. 1988. Software pipelining: An effective scheduling technique for VLIW machines. In Proceedings of the SIGPLAN 88 Conference on Programming Language Design and Implementation. 318-328.

C. Leiserson and J. Saxe. 1991. Retiming synchronous circuitry. Algorithmica 6, 1, 5-35.

A. Monot, N. Navet, F. Simonot, and B. Bavoux. 2010. Multicore scheduling in automotive ECUs. In Proceedings of the ERTSS. Toulouse, France.

L. Morel. 2005. Exploitation des structures régulières et des spécifications locales pour le developpement correct de systèmes réactifs de grande taille. Ph.D. Dissertation. Institut National Polytechnique de Grenoble.

S. Muchnick. 1997. Advanced Compiler Design and Implementation. Morgan Kaufman.

D.Potop-Butucaru, A. Azim, and S. Fischmeister. 2010. Semantics-preserving implementation of synchronous specifications over dynamic TDMA distributed architectures. In Proceedings of the EMSOFT. Scottsdale, AZ.

D. Potop-Butucaru, R. De Simone, Y. Sorel, and J.-P. Talpin. 2009. Clock-driven distributed real-time implementation of endochronous synchronous programs. In Proceedings of the 7th ACM International Conference on Embedded Software (EMSOFT'09). ACM, 147-156.

C. Pradalier, J. Hermosillo, C. Koike, C. Braillon, P. Bessière, and C. Laugier. 2005. The CyCab: A car-like robot navigating autonomously and safely among pedestrians. Robotics and Autonomous Systems 50, 1 , 51-68.

B. R. Rau. 1996. Iterative modulo scheduling. International Journal of Parallel Programming 24, 1, 3-64. 
B. R. Rau and C. D. Glaeser. 1981. Some scheduling techniques and an easily schedulable horizontal architecture for high performance scientific computing. In Proceedings of the 14th Annual Workshop on Microprogramming. IEEE.

B. R. Rau, M. Lee, P. P. Tirumalai, and M. S. Schlansker. 1992. Register allocation for software pipelined loops. In Proceedings of PLDI'92. San Francisco, CA.

J. Rushby. 2001. Bus architectures for safety-critical embedded systems. In Proceedings of EMSOFT'01 (LNCS), Vol. 2211. Tahoe City, CA.

M. Smelyanskyi, S. Mahlke, E. Davidson, and H.-H. Lee. 2003. Predicate-aware scheduling: A technique for reducing resource constraints. In Proceedings of the CGO. San Francisco, CA.

J. Wang and C. Eisenbeis. 1993. Decomposed software pipelining. http://hal.inria.fr/inria-00074834

N. J. Warter, D. M. Lavery, and W. W. Hwu. 1993. The benefit of predicated execution for software pipelining. In Proceedings of the HICSS-26 Conference. Houston, Texas.

H. Yang and S. Ha. 2009. Pipelined data parallel task mapping/scheduling technique for MPSoC. In Proceedings of the Design, Automation Test in Europe Conference Exhibition (DATE). Nice, France.

H.-S. Yun, J. Kim, and S.-M. Moon. 2003. Time optimal software pipelining of loops with control flows. International Journal of Parallel Programming 31, 5, 339-391.

J. Zalamea, J. Llosa, E. Ayguade, and M. Valero. 2004. Register constrained modulo scheduling. IEEE Transactions on Parallel and Distributed Systems 15, 5, 417-430.

W. Zheng, J. Chong, C. Pinello, S. Kanajan, and A. Sangiovanni-Vincentelli. 2005. Extensible and scalable time-triggered scheduling. In Proceedings of the ACSD. Saint-Malo, France.

Q. Zhuge, Z. Shao, and E. H. Sha. 2002. Optimal code size reduction for software-pipelined loops on DSP applications. In Proceedings of the International Conference on Parallel Processing.

Received September 2013; revised November 2013; accepted December 2013 